\section{Die biologische Abfallbehandlung als Beitrag zur Bioökonomiestrategie}

Die Menge an getrennt erfassten biogenen Abfällen aus dem kommunalen Bereich, wie „Biotonnenabfälle" oder Grünschnitt, stieg in Europa durch entsprechend zielgerichtete rechtliche Vorgaben (z. B. Ablagerungsverbot von biologisch abbaubaren Abfällen durch die EU-Deponierichtlinie, nationale Verordnungen zur getrennten Erfassung von biogenen Abfällen) in den letzten Jahren stetig an. In Österreich ist die bundesweite getrennte Sammlung von biogenen Siedlungsabfällen bereits seit dem Jahr 1992 obligatorisch, in Deutschland wurde im Zuge der Novellierung des Kreislaufwirtschafts- und Abfallgesetzes eine flächendeckende Bioabfallsammlung ab dem 1. Jänner 2015 beschlossen. Zusätzlich zu den kommunalen Mengen fallen spezifische Abfallfraktionen im gewerblichen und industriellen Bereich an. Diese branchenspezifischen biogenen Rückstände und Reststoffe, wie z. B. aus der Lebensmittelindustrie, werden heute schon zunehmend in innovativen kaskadischen Nutzungskonzepten effizient als Sekundärrohstoffe und Energieträger wieder eingesetzt und weitergenutzt. Die getrennt erfassten biogenen Siedlungsabfälle werden hingegen derzeit vorwiegend der „klassischen“ stofflichen Verwertungsschiene, der Kompostierung bzw. Teilmengen auch der anaeroben Verwertung, sprich der Biogasgewinnung, zugeführt.

Getrennt erfasste biogene Abfälle bieten prinzipiell auch ein hohes Potenzial als Inputmaterial für neuartige stoffliche Behandlungsverfahren und Nutzungssysteme, wie der Bioraffinerie. Dabei ist die effiziente Kaskadennutzung von biogenen Abfallstoffen und Biomasse die wesentliche Grundidee. Die Inputmaterialien sollen unter Anwendung verschiedener mechanischer, thermochemischer und biochemischer Verfahren möglichst vollständig in hochwertige stoffliche und energetische Produkte umgewandelt werden. Die Produktpalette reicht von Chemikalien, Werkstoffen, Futtermitteln bis hin zu Treibstoffen und Bioenergie. Das Konzept der Bioraffinerien stellt wiederum einen wichtigen Grundpfeiler des Bioökonomiekonzeptes dar, worunter generell die wissensbasierte Erzeugung und Nutzung biologischer Ressourcen verstanden wird, um Produkte, Verfahren und Dienstleistungen in allen wirtschaftlichen Sektoren im Rahmen eines zukunftsfähigen und nachhaltigen Wirtschaftssystems bereitzustellen. 2012 hat die Europäische Kommission in dem Strategie- und Maßnahmenplan „Innovation für nachhaltiges Wachstum: eine Bioökonomie für Europa“ auch Bio-Abfällen ein bedeutendes Potenzial als Alternative zu chemischen Düngemitteln und für die Umwandlung in Bioenergie zuerkannt. Das übergeordnete Ziel der Bioökonomie ist es, die wachsende Weltbevölkerung und Gesellschaft im 21. Jahrhundert mit Lebensmitteln, Industrieprodukten und Energie aus erneuerbaren Ressourcen, biologischen Abfällen und Reststoffen zu versorgen und damit auch die globale Ressourcenverknappung und den anthropogenen Treibhauseffekt zu bewältigen. Hervorzuheben ist hierbei, dass es bei der Nutzung des Potenzials an Abfällen zu keiner Konkurrenzsituation mit der Produktion von Lebensmitteln oder sonstigen landwirtschaftlich erzeugten Produkten kommt.

In der österreichischen Abfallwirtschaft wird schon seit mehr als 20 Jahren der biologischen Abfallverwertung und dem sorgsamen Umgang mit Bioressourcen ein entsprechender Stellenwert eingeräumt. Die Bioabfallbewirtschaftung erfolgt in Österreich im internationalen Vergleich auf einem hohen Niveau sowohl hinsichtlich Erfassungs- und Behandlungsquote wie auch Prozess- und Qualitätskontrolle der Outputprodukte, z. B. Kompost. Dieses Wissen und die langjährigen Erfahrungen können auch einen wesentlichen Beitrag zur Entwicklung der modernen Bioökonomiestrategie leisten.

Optimierungspotenzial hinsichtlich Erfassung und weiterer Verwertung besteht nach wie vor für den immer noch hohen biogenen Anteil in den gemischten Siedlungsabfällen (Restmüll). Selbst in den Vereinigten Staaten, wo bislang die hochtechnisierte Erfassung und energetische Nutzung von Deponiegas aus Siedlungsabfalldeponien, auf denen unvorbehandelte Abfälle mit hohem Deponiegasbildungspotenzial abgelagert werden, im Vordergrund stand, zeigte sich in den letzten Jahren vermehrt die Entwicklung

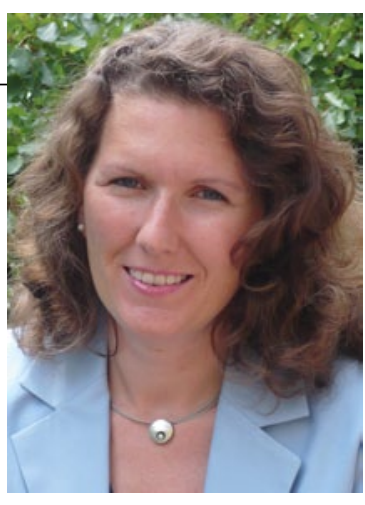

Priv.Doz. DI Dr. Marion Huber-Humer

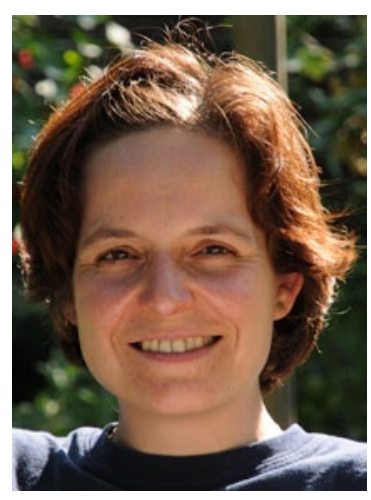

Univ.-Prof. Dr.-Ing. Anke Bockreis 
hin zur Abtrennung der organischen Anteile aus kommunalen Abfällen. Neue regulative Vorgaben, wie die Erfassung von $50 \%$ der Lebensmittelabfälle, was von der US EPA (oberste Umweltbehörde in den USA) als übergeordnetes Ziel gesetzt werden soll, bis hin zum angedachten Deponierungsverbot für organische Abfälle bis zum Jahr 2025 im Bundesstaat Kalifornien, verdeutlichen diesen Trend (Mitteilung von Bryan Staley, US Environmental Research \& Education Foundation, 2015). In der EU-Politik soll durch ein neues Strategiepapier "Towards a circular economy: A zero waste programme for Europe“ in Zukunft noch wesentlich stärker in Richtung Kreislaufwirtschaft und Minimierung der zu deponierenden Abfälle gelenkt werden, was verstärkt Einfluss auf den Umgang mit biogenen Abfällen und Rückständen haben wird.

Im vorliegenden Schwerpunktheft werden 4 Arbeiten zu aktuellen Themen und Fragestellungen der Bioabfallbewirtschaftung dargestellt. Die Arbeit von Kannengießer beschreibt einen Bioraffinerieansatz der Nutzung von biogenen Siedlungsabfällen zur Herstellung von biobasiertem Kraftstoff auf Basis unpolarer Carbonsäuren. Dazu wurde eine Machbarkeitsstudie durchgeführt, ob sich die biologischen Abfälle der Stadt Darmstadt zur Herstellung eines biobasierten Kraftstoffes eignen und welche Verfahrensschritte dafür kombiniert werden müssen.

Schneider et al. präsentieren in ihrer Arbeit ein neues Konzept zur Speiseresteaufbereitung mittels einer Vorbehandlung direkt am Entstehungsort. In einem Tanksystem wird ein lagerbares Substrat generiert, welches bedarfsgerecht sowie in Abhängigkeit von der Abfallmenge, vom Anfallort und von der abfallwirtschaftlichen Infrastruktur gesammelt und verwertet werden kann.

Die fachgerechte Bewirtschaftung von Bioabfällen kann zum Klimaschutz beitragen. Entscheidend ist allerdings, wie emissionsarm der Betrieb der Behandlungsanlagen geführt und wie effizient der Energieinhalt der biogenen Abfälle genutzt wird. Wie viel an klimarelevanten Methanemissionen während der biologischen Behandlung, sowohl bei der Kompostierung wie auch auf einer Biogasanlage, freigesetzt wird, untersuchten Hrad et al. in Feldstudien an österreichischen Anlagen. Bei diesen Untersuchungen kam erstmals eine innovative Open-path-Lasermessmethode in Kombination mit einer meteorologischen Ausbreitungsmodellierung zur Quantifizierung der Emissionsströme zum Einsatz.

Aber nicht nur Methanemissionen, sondern auch treibhausrelevante Lachgasemissionen können bei biologischen Abfallbehandlungsprozessen gebildet werden. Diesem Aspekt widmet sich der Artikel von Kraus et al., in dem der Einfluss unterschiedlicher Rotteparameter auf die Lachgasbildung und -freisetzung während der Intensivrottephase von Bioabfällen vorerst im Labormaßstab untersucht wurde. Auch hierbei kam ein innovatives Messgerät zum Einsatz, das die kontinuierliche Messung schon von geringsten Lachgaskonzentrationen (ppb-Bereich) ermöglicht.

Mit diesem Themenheft hoffen wir, dem Fachpublikum mit dem Einblick in aktuelle Forschungsarbeiten auch relevante und interessante Informationen bereit zu stellen und möchten uns an dieser Stelle bei allen bedanken, die an der Gestaltung und Erstellung dieses Heftes mitgewirkt haben.

Priv.Doz. DI Dr. M. Huber-Humer ( $₫)$

Institut für Abfallwirtschaft

Universität für Bodenkultur Wien

Muthgasse 107

1190 Wien, Österreich

E-Mail: marion.huber-humer@boku.ac.at

Univ.-Prof. Dr.-Ing. A. Bockreis

Institut für Infrastruktur

Universität Innsbruck

Technikerstraße 13a

6020 Innsbruck, Österreich

E-Mail: anke.bockreis@uibk.ac.at 